\title{
Square Root Module to Combat Dispersion-Induced Nonlinear Distortion in Radio-Over-Fiber Systems
}

\author{
Josep Prat, Member, IEEE, María C. Santos, and Mireia Omella
}

\begin{abstract}
Reduced dispersion-induced harmonics levels are reported for analogue radio-over-fiber systems by using a linearized receiver incorporating a memoryless electronic circuit with square root (SQRT)-like transfer function, and performing amplitude modulation (AM) at the transmitter. A practical implementation demonstrates the effectiveness of the AM-SQRT approach in linearizing the optical transmission system with respect to the conventional intensity modulation and direct detection system.
\end{abstract}

Index Terms-Adaptive equalization, dispersion equalization, optical fiber communications, radio-over-fiber (RoF).

\section{INTRODUCTION}

A PPLICATIONS of fiber antenna feeder solutions are growing at a breathless pace in a variety of wireless cellular, fixed broadband access, satellite, and radar systems. Transmission of microwave and millimeter-wave signals over optical fiber [radio-over-fiber ( $\mathrm{RoF})$ ] is an area of intense research activity mainly driven by the need of low-cost antenna terminals and the increase in operative frequencies.

In order to keep the remote antenna stations simple, the microwave carrier to be emitted through the radio channel is directly modulated over the optical wavelength [1]. This incurs in severe harmonic distortion caused by optical fiber chromatic dispersion whose degrading effect on the received signal scales as the square of the radio carrier [2]. To cope with it, a variety of equalization techniques both into the optical and into the electrical domain have been proposed [3]-[5], with great emphasis devoted recently to electronic equalization, due to higher adaptability and lower cost [5].

Being a linear effect on the optical field, low-cost linear equalizers could fully compensate for chromatic dispersion, but harmonic distortion generated after detection owing to the square-law characteristic of the photodiode significantly reduces the effectiveness of linear equalization [5].

We have proposed a square root (SQRT) transfer function module $(\mathrm{SRm})$ that placed after the photodiode compensates its square-law characteristic, improving the performance of linear equalizers [6]. The linearization capabilities of the SQRT module are analyzed here using the model of the dispersive fiber and the respective modulation and detection transfer functions for the conventional intensity (IM), as well as for amplitude modulation (AM) formats. The study considers the levels of second- and third-harmonic generated by each scheme, for a single radio-frequency carrier transmitted signal.

Manuscript received March 31, 2006; revised May 21, 2006. This work was supported in part by the EU FP6 NoE e-Photon/ONe, and in part by the Spanish MEC projects TEC2005-05160 and TEC2004-06194-C02.

The authors are with the Optical Communications Group, Universitat Politècnica de Catalunya, Barcelona 08034, Spain (e-mail: jprat@tsc.upc.edu).

Digital Object Identifier 10.1109/LPT.2006.881662

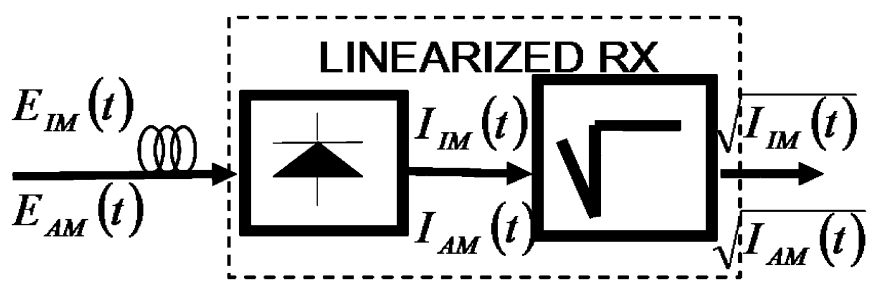

Fig. 1. Block diagram of the linearized receiver front-end.

The spectral characteristics obtained provide insight into the system linearization that improves the effectiveness of linear equalizers in digital systems, and are relevant to the performance of broadband multiple carrier RoF systems [3]. The proposed system is practically demonstrated.

The letter is organized as follows. In Section II, we describe the scheme of the optical transmission system with the linearized receiver and the mathematical model used to analyze it. We also give the harmonic levels for ideal IMs and optical field AMs. In Section III, we present the SQRT transfer function circuit and show simulation results for IM and AM modulations approximated by proper biasing of a Mach-Zehnder (MZ) modulator. In Section IV, we present the experimental setup and the measured results.

\section{THEORETICAL ANALYSIS}

The RoF receiver front-end of the system under study follows the scheme in Fig. 1. Both the cases of ideal IMs and optical field AMs are considered. The former is the usual case of conventional IM and direct detection (IM-DD) systems while in the latter the optical field envelope is proportional to the RF signal, being the instantaneous power its square. In order to assess the linearizing capabilities of the SQRT receiver, we have computed the level of harmonics generated when a pure microwave carrier is transmitted. The normalized fields launched into the fiber by ideal modulators, with zero chirp are given, respectively, by the expressions

$$
\begin{aligned}
E_{\mathrm{IM}}(t) & =\sqrt{1+m_{i} \cos (w t)} e^{j w_{o} t} \\
E_{\mathrm{AM}}(t) & =\left[1+m_{a} \cos (w t)\right] e^{j w_{o} t}
\end{aligned}
$$

with $m_{i}, m_{a}$ the respective modulation indexes and $w$ and $w_{o}$, respectively, the microwave carrier and the optical frequency in radians per second. Even when the modulation index is a more common parameter in the context of RoF systems, in order to obtain AM and IM signals with comparable modulation depths in terms of sidebands level relative to carrier, the $m_{i}, m_{a}$ are chosen in each case to maintain a given optical extinction ratio, $\mathrm{ER}=20 \log \left(E_{\max } / E_{\min }\right)$. 


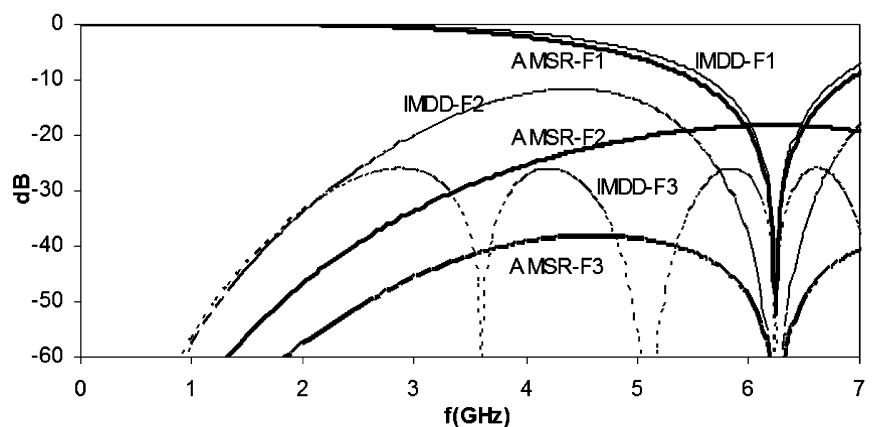

Fig. 2. Fundamental and harmonic power levels (normalized to low-frequency F1) of the IM-DD and AM-SR systems versus the modulating frequency, for $100 \mathrm{Km}$ of SSMF and $\mathrm{ER}=10 \mathrm{~dB}$.

The low-pass frequency transfer function for a fiber with dispersion parameter $D$ and length $L$ is [2]

$$
H(f)=\exp \left(-j \frac{\pi}{c} \lambda^{2} f^{2} D L\right)
$$

where $\lambda$ is the optical wavelength and $c$ the speed of light in vacuum.

The optical field impinging on the photodetector is the convolution of the input field with the fiber impulse response $h(t)$, the inverse Fourier transform of $H(f)$. The photodiode performs a modulus squared operation on the optical field that causes optical linear effects to become nonlinear. The normalized photodetected current is obtained as

$$
I_{D}(t)=|E(t) * h(t)|^{2}
$$

and neglecting high frequency components outside the detectors electrical bandwidth. For the amplitude modulated signal (AM-DD system), in terms of the chromatic dispersion index $\chi=\pi \lambda^{2} f^{2} D L / c$, it gives

$$
I_{\mathrm{AM}}(t)=1+\frac{m_{a}^{2}}{2}+2 m_{a} \cos (\chi) \cos (w t)+\frac{m_{a}^{2}}{2} \cos (2 w t) .
$$

Linear dispersion compensation of the $\cos (\chi)$ factor at the fundamental frequency $(\mathrm{F} 1)$ is hindered by the term at the secondharmonic frequency (F2), which is due to the photodiode inherent nonlinearity.

If now, we take the SQRT operation (Fig. 1), we obtain, up to order $m^{3}$, and labelled AM-SR system

$$
\begin{aligned}
\sqrt{I_{\mathrm{AM}}(t)} \approx & +\frac{m_{a}^{2}}{4} \sin ^{2}(\chi)+m_{a} \cos (\chi) \cos (w t) \\
& +\frac{m_{a}^{2}}{4} \sin ^{2}(\chi) \cos (2 w t) \\
& -\frac{m_{a}^{3}}{8} \cos (\chi) \sin ^{2}(\chi) \cos (3 w t) .
\end{aligned}
$$

We get a cancellation of F2 at low frequencies which provides an extended frequency margin for a given tolerable level of nonlinear distortion. This is shown in Fig. 2 for $100 \mathrm{Km}$ of fiber with $D=16 \mathrm{ps} /(\mathrm{nm} \cdot \mathrm{Km})$. Note that the plots are normalized to the fundamental power (F1) at low frequencies.

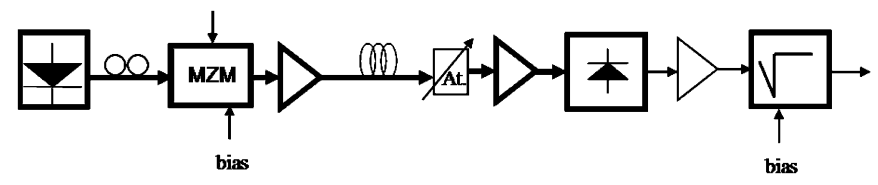

Fig. 3. Experimental setup.

For conventional IM-DD systems the photodiode current, up to $m^{3}$ terms, is

$$
\begin{aligned}
I_{\mathrm{IM}}(t) \approx & 1+m_{i} \cos (\chi) \cos (w t) \\
& +\frac{m_{i}^{2}}{4} \sin ^{2}(2 \chi) \cos (2 w t) \\
& -\frac{m_{i}^{3}}{8} \cos (3 \chi) \sin ^{2}(3 \chi) \cos (3 w t) .
\end{aligned}
$$

When comparing (6) and (5), we see they feature the same basic dependences with $\chi$, but in (6) respective factors 2 and 3 are added for the F2 and F3. Therefore, with the AM-SR system, we could travel twice the distance to have the same level of F2 and three times the distance in the case of F3, or else, for the same distance, we could increase the modulation frequency by a factor $\sqrt{2}$ and $\sqrt{3}$, respectively.

The simulation results, shown in Fig. 2 for a TX extinction ratio of $10 \mathrm{~dB}$, are slightly better than those predicted by (5) and (6), and confirm the linearizing capabilities of the SRm into a radio-frequency band. As a quantitative example, for a $100-\mathrm{Km}$ link with $D=16 \mathrm{ps} /(\mathrm{nm} \cdot \mathrm{Km})$ and considering a maximum tolerance of $40 \mathrm{~dB}$ of harmonics level, we could extend the operative band from $1.67 \mathrm{GHz}$ for the conventional IM-DD system, up to $2.45 \mathrm{GHz}$ with the AM-SR, limited by the F2 reaching the 40-dB level, or even up to $3.81 \mathrm{GHz}$ where the $\mathrm{F} 3$ gets that level. This represents a little bit more than the expected respective $\sqrt{2}$ and $\sqrt{3}$ improvement factors. Note that the results depend on the frequency and distance via the chromatic dispersion index $\chi$, and therefore, the results for $100 \mathrm{Km}$ and $7 \mathrm{GHz}$ apply for a different distance-frequency range, for example $1 \mathrm{Km}$ and $70 \mathrm{GHz}$. Qualitatively, the same frequency characteristics are obtained with lower ER values, more commonplace in practical RoF systems, with of course lower harmonic levels, well approximated by (5) and (6). Taking ER $=2.5 \mathrm{~dB}$, thresholds are $-23.1 \mathrm{~dB}$ for the IM-DD-F2 case, and $-28.9 \mathrm{~dB}$ for the AMSR-F2, with a frequency increase from $2.2 \mathrm{GHz}$ up to $3.7 \mathrm{GHz}$. As for third-harmonic levels, they reach $-48.5 \mathrm{~dB}$ in IM-DD and $-60 \mathrm{~dB}$ in AMSR systems.

\section{PRACTICAL DESIGN}

In the practical setup (Fig. 3), the IM modulation is obtained by biasing the $\mathrm{MZ}$ modulator around the quadrature $\left(90^{\circ}\right)$ point, as it is common practice, while for the AM a bias closer to the zero transmission point $\left(135^{\circ}\right)$, is used. Around this point, the modulator input-output characteristic (voltage-optical power) tends to a quadratic function, and thus it is approximately linear with the optical field amplitude.

The SQRT nonlinear equalizer module has been implemented by analogue electronics, using discrete Schottky diodes. The resulting circuit can be modelled as shown in Fig. 4. The current-voltage transfer function yields a lower than $10 \%$ fitting error with respect to the ideal SQRT, within the useful current margin.

Simulations have been also correspondingly carried out for the practical setup in Fig. 3, taking the MZ transfer function 


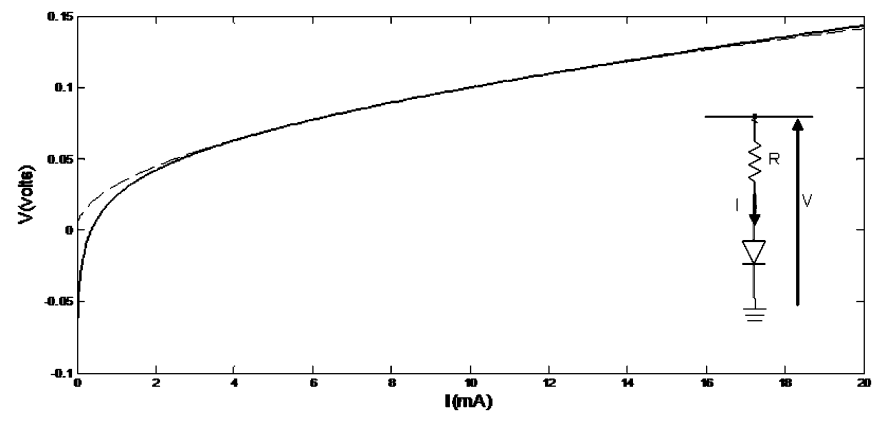

Fig. 4. SQRT circuit model voltage-current characteristic (solid line) and ideal SQRT function (dashed line).

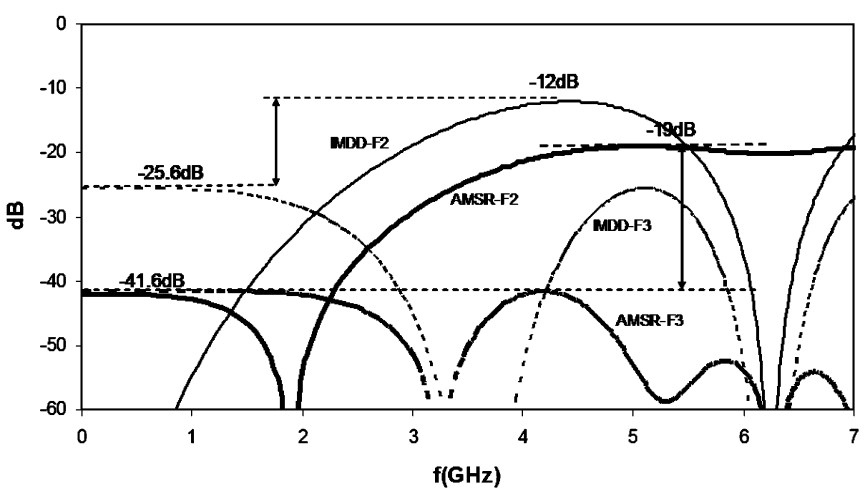

Fig. 5. Harmonic power levels (normalized to low-frequency F1) obtained by simulation of the practical setup, Fig. 3, with the diode circuit model in Fig. 4, 100-km SSMF, ER $=10 \mathrm{~dB}$, and proper biasing of the MZ; IM: $90^{\circ}$, AM: $135^{\circ}$.

and the nonlinear memoryless input-output relationship of the diode circuit model (Fig. 4). The resulting nonlinear harmonic power levels are shown in Fig. 5, comparing the IM-like system (MZ at quadrature and DD) to the AM-SR-like system (MZ biased at $135^{\circ}$ and receiver with SQRT circuit). We observe that the results are very similar to the ideal cases. The greater differences are found at low frequencies which characterize the back-to-back intrinsic system nonlinear behaviour due to the nonlinear transfer functions of modulator and detector and where the AM-SR system yields a higher F2. The low-frequency levels are in any case well below the passband values and should not entail a significant reduction of performance. More importantly, at high frequencies, the AM-SR approach maintains its linearity advantage in front of the conventional system.

\section{MEASUREMENTS}

An analogue optical transmission system of $100 \mathrm{Km}$ of standard single-mode fiber $(\mathrm{SSMF})(D=16 \mathrm{ps} /(\mathrm{nm} \cdot \mathrm{Km}))$ operating at $1550 \mathrm{~nm}$ has been set up (scheme in Fig. 3), and the harmonics power for the same $\mathrm{ER}=10 \mathrm{~dB}$ in a margin of electrical modulating frequencies from $200 \mathrm{MHz}$ up to $7 \mathrm{GHz}$ has been measured (Fig. 6). The measured levels present the same basic dependence with frequency as that predicted by simulations and confirm the linearisation capabilities of the AM-SR approach. At frequencies above $2 \mathrm{GHz}$, the harmonic levels of the proposed AM-SR system are well below those of conventional IM-DD. The nonlinearity reduction in the worst case

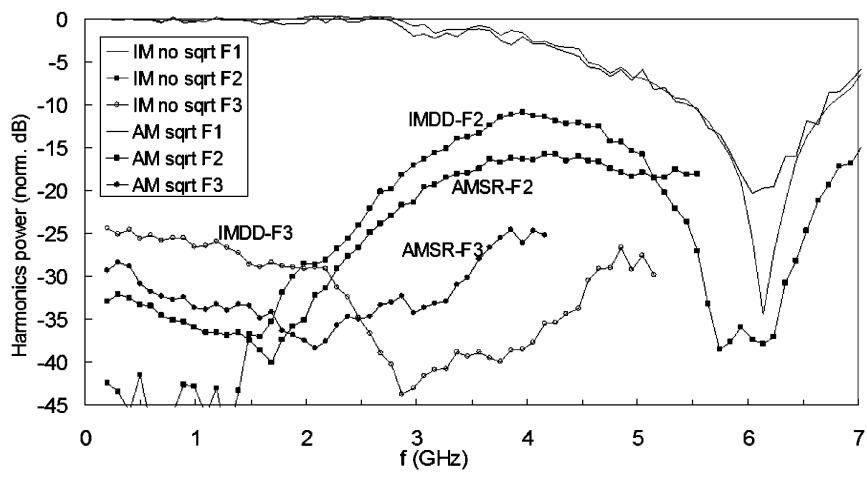

Fig. 6. Measured harmonic power levels (F2 and F3) normalized to low-frequency $\mathrm{F} 1$ power, for a $100-\mathrm{Km} \mathrm{SSMF}$ and $\mathrm{ER}=10 \mathrm{~dB}$, as a function of modulating frequency.

margin (around $4 \mathrm{GHz}$ of fundamental) is about $5 \mathrm{~dB}$. The lowcost SQRT circuit is bandwidth-limited, causing to work with very low powers at the higher frequencies ( $4 \mathrm{GHz}$ of $\mathrm{F} 3$ means operation at $12 \mathrm{GHz}$ ). Normalization at these frequencies produces a noise amplification effect and some deviation from simulated results in Fig. 6. Still, the predicted linearity improvement in the passband frequencies is seen.

\section{CONCLUSION}

We have analyzed the performance of an SQRT transfer function module placed after the photodiode detector in a single carrier analogue fiber system. Our analytical results, validated through numerical simulations and measurements, show that this linearized receiver allows for effective reduction of harmonics when the signal is amplitude modulated at the transmitter side (AM-SR approach). The simulations predict respective approximate factors $\sqrt{2}$ and $\sqrt{3}$ of operative frequency increase with respect to conventional IM-DD systems (factors 2 and 3 in distance range) for the same second- and third-harmonic levels, thus holding promise to extend the reach of present RoF systems as well as for high-bit rate digital transmission with electronic linear dispersion compensation.

\section{REFERENCES}

[1] T. Koonen, A. Ng'oma, M. García-Larrode, F. Huijskens, I. M. Tafur-Monroy, and G.-D. Khoe, "Novel cost-efficient techniques for microwave signal delivery in fibre-wireless networks," in Proc. ECOC'04, 2004, vol. 5, pp. 120-125, Paper Th1.1.1.

[2] U. Gliese, S. Norskov, and T. N. Nielsen, "Chromatic dispersion in fiber-optic microwave and millimeter-wave links," IEEE Trans. Microw. Theory Tech., vol. 44, no. 10, pt. 1, pp. 1716-1724, Oct. 1996.

[3] J. Marti, D. Pastor, M. Tórtola, J. Capmany, and A. Montero, "On the use of tapered linearly chirped gratings as dispersion-induced distortion equalizers in scm systems," J. Lightw. Technol., vol. 15, no. 2, pp. 179-187, Feb. 1997.

[4] D. Stahl, P. J. Winzer, C. R. Doerr, and S. Chandrasekhar, "Extending the chromatic dispersion tolerance by optical equalization at $43 \mathrm{~Gb} / \mathrm{s}$," in Proc. OFC 2004, Los Angeles, CA, vol. 2, Paper ThU5.

[5] V. Curri, R. Gaudino, A. Napoli, and P. Poggiolini, "Electronic equalization for advanced modulation formats in dispersion-limited systems," IEEE Photon. Technol. Lett., vol. 16, no. 11, pp. 2556-2558, Nov. 2004

[6] J. Prat, A. Napoli, J. M. Gené, M. Omella, P. Poggiolini, and V. Curri, "Square root strategy: A novel method to linearise an optical communications system with linear equalizers," in Proc. ECOC 2005, Glasgow, U.K., 2005, Paper We4.P.106. 